\title{
Model Update Particle Filter for Multiple Objects Detection and Tracking
}

\author{
Yunji Zhao $^{1}{ }^{*}$, Hailong Pei ${ }^{2}$ \\ ${ }^{1}$ School of Electrical Engineering and Automation, Henan Polytechnic University, \\ Jiaozuo,Henan,454000, China. \\ E-mail: auzhaoyunji@163.com \\ ${ }^{2}$ College of Automation Science and Engineering, South China University of Technology, \\ Guangzhou, 510640,China. \\ E-mail:auhlpei@scut.edu.cn \\ Received 30 November 2011 \\ Accepted 19 June 2012
}

\begin{abstract}
Multiple objects tracking is a challenging task. This article presents an algorithm which can detect and track multiple objects, and update target model automatically. The contributions of this paper as follow: Firstly,we also use color histogram $(\mathrm{CH})$ and histogram of orientated gradients(HOG) to represent the objects, model update is realized by kalman filter and gaussian model; secondly we use Gaussian Mixture Model(GMM) and Bhattacharyya distance to detect object appearance. Particle filter with combined features and model update mechanism can improve tracking results. Experiments on video sequences demonstrate that the method presented in this paper can realize multiple objects detection and tracking.
\end{abstract}

Keywords: Color Histogram, Histogram of Oriented Gradients, Particle Filter, Gaussian Mixture Model

\section{Introduction}

Visual object tracking is an important task in the field of computer vision. It is widely used in motionbased recognition ${ }^{1}$, automated surveillance, humancomputer interaction ${ }^{2}$, vehicle navigation and traffic monitoring ${ }^{3}$. Object description mainly depends on the image resolution and available computational resources. Yilmaz et al. ${ }^{3}$ gave an important issues related to tracking including the use of appropriate image features, selection of motion model and objects detection.

Color is one of the most widely used appropriate image features in object tracking as its effectiveness and efficiency ${ }^{4,5}$. $\mathrm{CH}$ is robust to scale variation, rotation and small changes of form. So it can be used in many tracking situations,such as partial occlusion and the form of object is non-rigidity. However $\mathrm{CH}$ is subject to similar color of its background and illumination changes. In view of the shortcomings of $\mathrm{CH}$ other characters,such as texture and contour are employed to represent the object ${ }^{6}$. Spatial distribution of $\mathrm{CH}$ is also used to represent the object ${ }^{7}$. Recently,histogram of oriented gradients(HOG) is employed to detect and track object ${ }^{8,9,10}$. Navneet Dalal and Bill Triggs ${ }^{8}$ studied the issue of feature sets for human detection, showing that locally normalized HOG descriptors provided excellent performance relative to other existing feature sets including wavelets ${ }^{11}$. HOG can represent the edges and local contours. So it is not subject to color variation. However HOG also has shortcomings simi-

*Corresponding author.Email:auzhaoyunji@gmail.com or auzhaoyunji@163.com 
lar to other contour and texture features,such as orientation sensitive and not effectively describing the objects or backgrounds with large smooth regions. According to the drawbacks of HOG when objects rotate HOG should be updated in time. We integrate $\mathrm{CH}$ and $\mathrm{HOG}$ to achieve mutual complementarities of the two types of features. However $\mathrm{CH}$ together with HOG can be applied to deal with some appearance and shape changes to achieve robust tracking performance, model update should be taken into consideration. As in Ref. 12 we also use kalman filter to do model update.

Deterministic methods localize the tracked object in each frame by iteratively searching for a region which maximizes the similarity measure between the candidate and the object. Comaniciu et al. ${ }^{13}$ employed mean shift algorithm to do object tracking. Mean shift algorithm is computationally efficient. However mean shift algorithm may converges to a local maximum, they are also sensitive to background distracts, occlusions, and objects moving quikly. Among various of stochastic methods, Particle filter ${ }^{9,10}$ is widely used and makes the tracking system robust. Hanzi Wang et al. ${ }^{14}$ proposed a similarity measure based on Spatial-color mixture of Gaussians appearance model for particle filters. Peter Dunne and Bogdan Matuszewski ${ }^{15}$ considered the choice of particle filter dissimilarity distance measures and likelihood functions in the context of object tracking in grey scale video. Katja Nummiaro et al. ${ }^{16}$ presented the integration of color distributions into particle filter, which has been typically used in combination with edge-based image features. To effectively abate the influence of particle degeneracy and improve the robustness of visual tracking Hongwei Li et al. ${ }^{17}$ proposed a novel visual tracking algorithm using particle filter with Gaussian process regression and re-sampling techniques. In Ref. 18 a robust visual object tracking scheme is proposed that exploits the dynamics of object shape and appearance similarity. The method uses a particle filter where a multi-mode anisotropic mean shift is embedded to improve the initial particles. Budi Sugandi et al. ${ }^{19}$ proposed a method for multiple object tracking based on color features. Whist Jacek Czyz et al. ${ }^{20}$ proposed that target detec- tion and deletion are embedded in the color particle filter without relaying on an external track initialization and cancelation algorithm to track multiple targets. In Ref. 21 the author also used the color particle filter to realized object tracking, but the global histogram was replaced by a rough spatial layout with multi-part color modeling, the author also used background color model in multiple objects tracking. Not all of the tracking system based on color particle filter employe model update. In Ref. 10 and Ref. 19 the authors used different methods to realize model update. In Ref. 10 the author introduced switching probabilistic principal component analysis model to update the templates while in Ref. 19 color histogram was replace by the sum color histogram of target and estimate. Some of color particle filter introduce model update as Ref. 19, but this type of methods are subject to tracking drift. We introduce kalman filter to do model update. In Ref. 20 the author proposed multiple objects tracking,the object was detected based on Bayesian estimation. In this article we introduce GMM and Bhattacharyya distance to do the object detection.

The paper is organized as follows. In section 2 $\mathrm{CHOG}$ is reviewed. Model update mechanism based on kalman filter and gaussian model is introduced. In section 3 object detection and occlusion handling are presented. Experiments is presented in section 4 and the conclusion is in section 5 .

\section{Features Extraction and Object Tracking with Model Update}

\subsection{Features Extraction}

In this section object features extraction and model update in particle filter tracking system is presented. Object features described by single cue do not work well all the time. Many researches have employed multiple cues for tracking system ${ }^{9,10,14,15,16}$. In this article we introduce Hue-Saturation-Value(HSV) $\mathrm{CH}$ to represent the color information and the HOG to represent the shape information. Hue and Saturation color histogram is batter than RGB color histogram in describing the color features of object with illumination change. In this article we use 
HSV color histogram,each of them is composed of $N_{h} \times N_{s}+N_{v}=8 \times 8+8$ bins.

HOG can describe the shape information of objects. HOG is calculated in gray-scale space as Ref. 9 described. As SIFT ${ }^{22}$ (Scale Invariant Feature Transform) we adopt the similar method to obtain the HOG, whilst we introduce the weighting function. To increase the reliability of the HOG when boundary pixels belong to the background,smaller weights are assigned to the pixels further away from the region center. The weighting function as:

$$
w=\exp \left(-(i * i+j * j) / 2 * \delta^{2}\right)
$$

where $i$ and $j$ is the coordinate of the pixels depending on the origin which is the center of the computational domain. $\delta$ is the same as the ones which is used to smooth the image to avoid the affection of noise. According to the weights the HOG of the region will be obtained as follow:

$$
B_{y}^{u}=\sum_{i=1}^{I} w\left(x_{i}\right) \boldsymbol{\delta}\left(b\left(x_{i}\right)-u\right)
$$

where $B_{y}^{u}$ represents the magnitude of $u$ directions in region $y . I$ is the number pixels in the region of $y, \delta$ is the Kronecker delta function, $b\left(x_{i}\right)$ is the function of HOG computed at the pixel $x_{i}$. Fig. 1 shows the CHOG of different object. To deal with the object rotation,dominant orientation method is adopted as Ref. 22. Coarse orientation histogram which is composed of 8 bins as Fig. 1 shows. It is used to rotate the HOG to ensure that the instantaneous dominant orientation is consistent with the reference. After that HOG composed of 72 bins is calculated as Ref. 9.

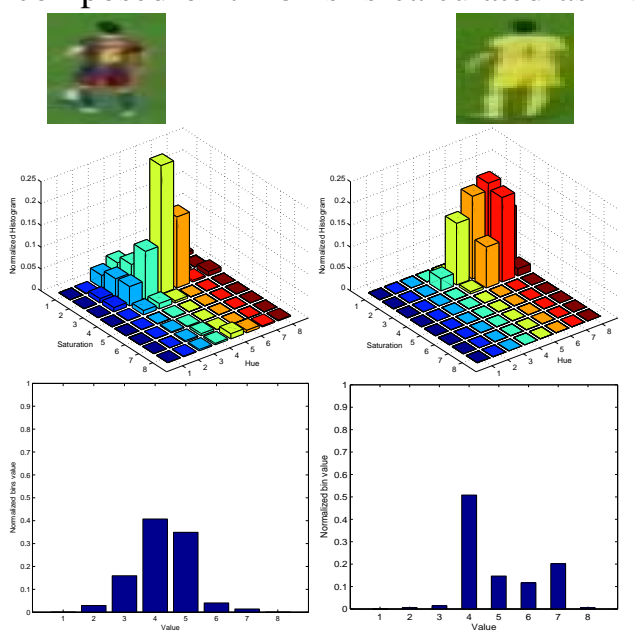

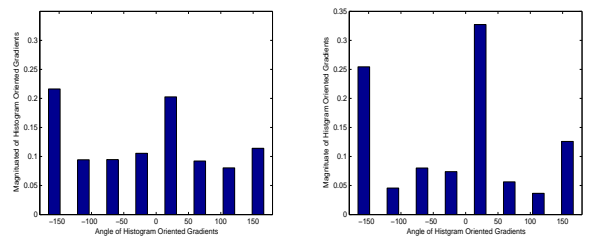

Fig. 1. CHOG of different players.

\subsection{Model Update}

Adaptive model update mechanism is very important for real-time object tracking. Ning Song Peng ${ }^{23}$ proposed a novel method to achieve adaptive model update by kalman filter. As Ref. 23 described we introduce kalman filter to obtain the optimal estimate of object model,whilst the HOG model is updated based on Gaussian Model. In the frame of kalman filter when the object is tracked the description of it is regarded as observation model while the description of object used in process of tracking is called current model. Tread off between these models is made to obtain the candidate model for next frame tracking. Candidate model is not always suitable, so a robust criterion to decide whether the candidate model should be accepted or not is necessary. Considering two consecutive frames $i-1$ and $i$, for each bin $b$, model update equations according to kalman filter as follows: state prediction equations:

$$
\widehat{p}_{b}^{i \mid i-1}=\widehat{p}_{b}^{i-1}
$$

Variance of prediction error:

$$
q_{b}^{i \mid i-1}=q_{b}^{i-1}+\delta_{w}^{2}
$$

where the superscript $i \mid i-1$ denotes the prediction of the frame $i$ based on information of the previous frame $i-1$. Gain equation:

$$
m_{b}^{i}=\frac{q_{b}^{i \mid i-1}}{q_{b}^{i \mid i-1}+\delta_{v}^{2}}
$$

where $\delta_{v}^{2}$ is the variance of measurement noise. Optimal estimate of $p_{b}^{i}$ as

$$
\widehat{p}_{b}^{i}=\hat{p}_{b}^{i \mid i-1}+m_{b}^{i}\left(o_{b}^{i}-\hat{p}_{b}^{i \mid i-1}\right)
$$


Variance of filtering error:

$$
q_{b}^{i}=\left(1-m_{b}^{i}\right) q_{b}^{i \mid i-1}
$$

Filtering residual:

$$
r_{b}^{i}=o_{b}^{i}-\hat{p}_{b}^{i \mid i-1}
$$

At frame $i$ the variance of filtering residuals of all the bins over the last $L$ frames:

$$
\left(\bar{r}^{i}\right)^{2}=\frac{1}{m L} \sum_{l=i-L+1}^{i} \sum_{b=1}^{m}\left(o_{b}^{l}-\hat{p}_{b}^{l \mid l-1}\right)^{2}
$$

$\delta_{w}^{2}$ is estimated:

$$
\delta_{w}^{2}=\left(\bar{r}^{i}\right)^{2}-\delta_{v}^{2}-\frac{1}{m} \sum_{b=1}^{m} q_{b}^{i-1}
$$

Initial parameters can be obtained as follow ${ }^{24}$ :

$$
\delta_{v}^{2}=0.5(\bar{r})^{2}, \delta_{w}^{2}=0, q_{u}^{0}=0.5(\bar{r})^{2}
$$

In order to obtain an more optimal candidate significance level $\alpha$ was introduced ${ }^{23}$. Filtering residuals was supposed as a normal distribution $R \sim N\left(\mu, \delta^{2}\right)$. $t$ distribution of filtering residuals is described as follow:

$$
t=\frac{\bar{r}-\mu_{0}}{s / \sqrt{N}}
$$

where $s$ is the square root of the standard deviation, $N$ is the sample number of filtering residuals, $\left\{r_{i}\right\}_{i=1,2, \ldots N}$. Null hypothesis $H_{0}: \mu \leqslant \mu_{0}$ and alternative hypothesis $H_{1}: \mu>\mu_{0}$ is supposed and they will be determined by the significance level $\alpha$. When $H_{0}$ is true, the $t$ distribution is approximated by $t(N-1)$ distribution. According to conditional probability:

$$
p\left(\text { refuse } H_{0} \mid H_{0} \text { is true }\right)=p\left(\frac{\bar{r}-\mu_{0}}{s / \sqrt{N}} \geqslant c r\right)=\alpha
$$

$c r=t_{\alpha}(N-1)$ is obtained and the critical region is determined:

$$
t=\frac{\bar{r}-\mu_{0}}{s / \sqrt{N}} \geqslant c r
$$

where $\mu_{0}$ is set to $\bar{r}^{i}$ according to Eq.(9). So the optimal estimation of color histogram as:

$$
\widehat{p}_{b}^{i}= \begin{cases}\widehat{p}_{b}^{i \mid i-1}+m_{b}^{i}\left(o_{b}^{i}-\widehat{p}_{b}^{i \mid i-1}\right) & \text { if accept } \mathrm{H}_{0} \\ \widehat{p}_{b}^{i-1} & \text { if accept } \mathrm{H}_{1}\end{cases}
$$

The HOG of object should be also updated. In view of the HOG is eight dimension and the sample numbers with significance level $\alpha=0.1$ should be more than eight,we introduce gaussian model to update the HOG of the object. HOG is represented as $\left\{B_{i}\right\}_{i=1,2, \ldots 8}$. We suppose each bins as a normal distribution, $B_{i} \sim N\left(\mu, \delta^{2}\right) . B_{i}$ is regard as a time series from the frame $t_{0}$ to $t_{k}$. According to this the average $\mu$ and covariance $\delta^{2}$ will be obtained. In order to avoid the affection of noise the optimal estimation of HOG as follows:

$$
B_{i}= \begin{cases}\beta \mu+(1-\beta) B_{i} & \text { if } B_{i} \leqslant 3 \delta \\ \mu & \text { if } \mathrm{B}_{i}>3 \delta\end{cases}
$$

where $\beta$ is the learning rate which determines the rate of object updating, we always choose it as $\beta=$ 0.1 . Up to now the features of the object composted of color histogram and histogram oriented gradients are achieved.

\subsection{Object Tracking with Model Update}

Particle filter is specifically designed for sequential Bayesian estimation when the system is non-linear and non-gaussian. We employ the Bhattacharyya distance to update the priori distribution obtained by particle filter. Each sample of the distribution represents the object as $X(x, y, s, x p, y p, s p, w, h, x 0, y 0)$, where $x, y$ specify the current location of the object, $x p, y p$ specify the previous location of the object, $s$, $s p$ specify the current scale and the previous scale of the object, $w, h$ represents the width and height of the object, $x 0, y 0$ represents the original $x$ and $y$ coordinate.

The sample set is propagated through secondorder autoregressive dynamics model.

$$
X_{t}=A X_{t-1}+\omega_{t-1}
$$

where $\omega_{t-1}$ is a multivariate Gaussian random variable, $A$ defines the state estimate matrix. In order to 
make the computation of weight easily,each weight is defined by $w_{t}(k)=c \exp (-\lambda \rho)$, where $w_{t}(k)$ represents the $k t h$ particles weight, after all the weights of particles obtained each of weights normalized, $\rho$ is the Bhattacharyya distance between the features of tracking object and the features of object moved randomly in a certain region. After that the $N$ particles are obtained with normalized weights. The mechanism of model update particle filter is introduced correctly as follow:

- Choose a tracking object in a vision sequence and obtain the color histogram and histogram oriented gradients descriptions of it $q_{0}$.

- Move the object randomly in certain regions $N$ times and achieve the descriptions of them by means of Eq.(17). According to Eq.(1) and the Bhattacharyya distance assign the weights to each of them. Sample set $S_{1}$ is obtained which include $N$ particles.

- Estimate the mean state of set $S_{1}$ and use the object description component to update the object model $q_{1}$ by means of kalman filter and gaussian model.

- Re-sample in particles set $S_{1}$ obtains new particles set $S_{2}$

- Do while the step 2, 3, 4 to realize the model update object tracking based on particle filter

Fig. 2 shows the tracking effect of the tracker based on CHOG and CHOG with model update. The footballer is chose as the object when the footballer turn around the model composed of $\mathrm{CH}$ and HOG may be changed. The changed model may affect the tracking effect as Fig.2 shows. This experiments testify that model update is necessary in some conditions.
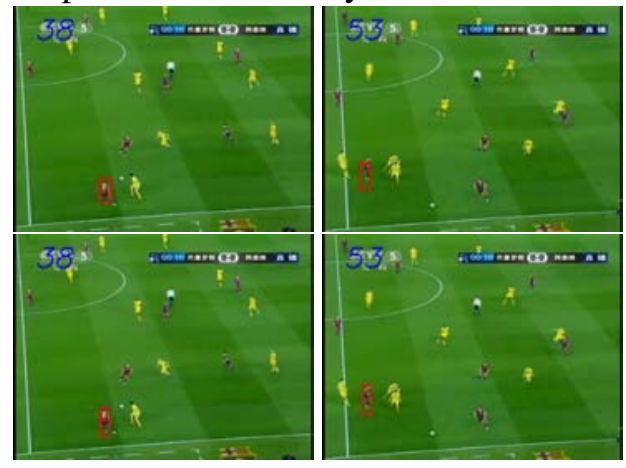
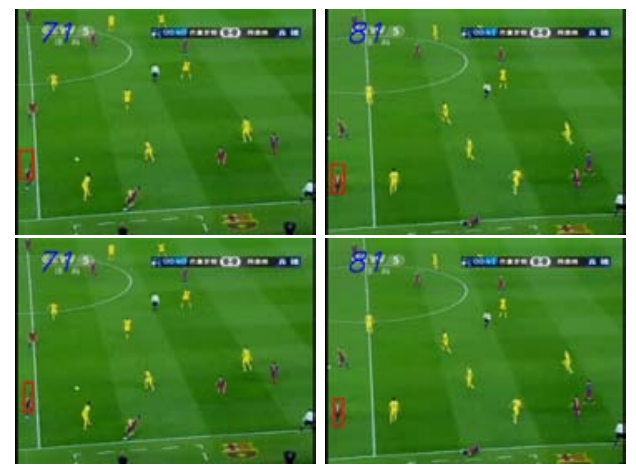

Fig. 2. Comparison between the CHOG algorithm and model update CHOG algorithm, the above results are CHOG and the bellows are model update CHOG algorithm.

\section{Object Detection and Occlusion Handling}

\subsection{Objects Detection}

Gaussian model is widely used in target tracking $25,26,27,28,29$. In Ref. 27 and Ref. 28 the gaussian model is used to approximates the posterior distributions under the concept of particle filter. So the Gaussian particle filter is similar to Gaussian filters as the extended Kalman filter and its variants. It is shown that under the gaussian assumption, the Gaussian particle filter is asymptotically optimal in the number of particles and has much-improved performance and versatility over other Gaussian filters, especially nontrivial nonlinearities. In Ref. 25 and Ref. 26 the Gaussian model is used to model the foreground and background. In Ref. 25 the background pixels are modeled as a single gaussian while the foreground pixels are modeled as a mixture gaussian. In Ref. 26 the background and foreground model are similar to Ref. 25, but it presents a method which improves the adaptive background mixture model to distinguish between moving shadows and moving objects. In Ref. 29 a spatial-color mixture of gaussian appearance model for particle filters is proposed. This method improves the popular similarity measure based on color histogram because it considers not only the colors in a region but also the spatial layout of colors. The experiments of the method which is spatial-color mixture of gaus- 
sian appearance model for particle filters show that it can successfully track objects in many difficult situations.

In this paper we introduce gaussian mixture model(GMM) to detect the tracking object as Ref. 25 and Ref. 26 used. Fig. 3 shows the footballers may be locate in the sequence. The locations, area and number of objects are determined by Blob algorithm ${ }^{30}$. As Fig. 3 we need to calculate the kernel color histogram in the bright region as Ref. 16 and the HOG according to Eq.(1) and Eq.(2). After we obtain the locations of objects we use Bhattacharyya distance between the features of object and the one in this region to identify which is the real one we want to track by setting threshold. In the experiments we set the threshold $T=0.1$ because sometimes the histogram is subject to the footballers turn around in football sequence. If the Bhattacharyya distance is smaller than $T$ the object is not we want to track. When the objects are detected we use the CHOG particle filter to track. Fig.4 shows the objects detection under the fixed angle of view.

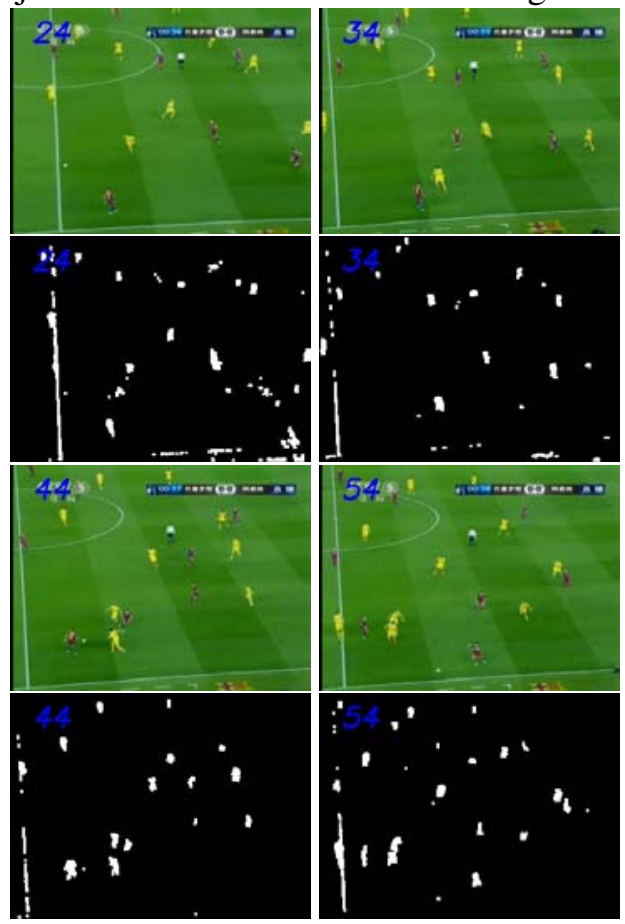

Fig. 3. Locations of objects detected by GMM.

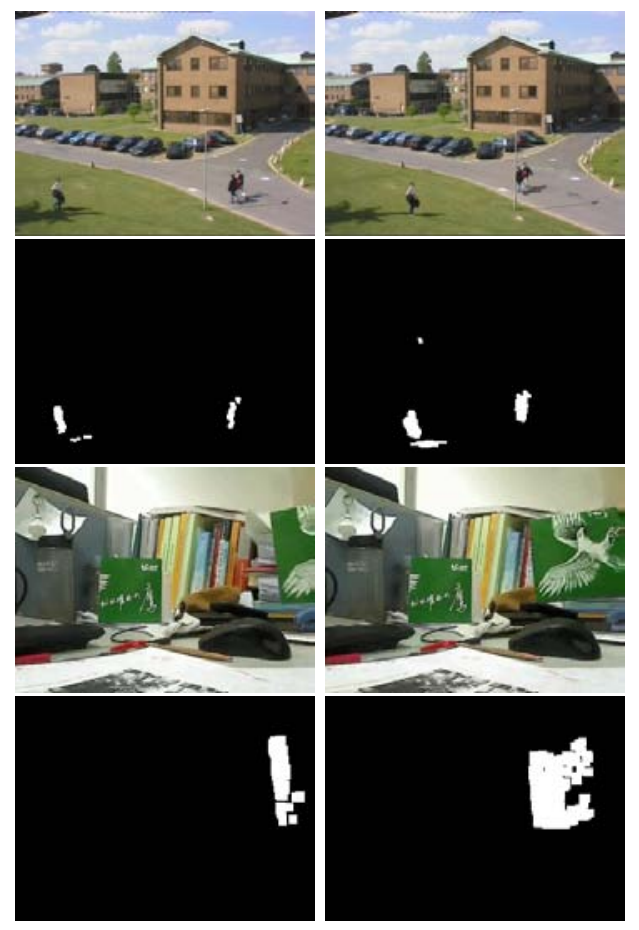

Fig. 4. Objects detection under the fixed angle of view.

The method of GMM used to detect objects is batter than Ref. 20 in which as Fig. 3 shows. Person $\mathrm{B}$ entering the scene in frame 50 is detected in frame 60. In the frame of GMM when the object appearance in the scene it is detected not far away from the next frame.

\subsection{Occlusion Handling}

Occlusions may cause the model update tracker failure. It is severe in model update particle filters because of the inaccuracies in updating the position, size and the features of the tracked objects. So the object's estimated position and size may be adapted to the area not occluded. Moreover the features of the object may be updated by incorrect values, which lead to target loss after a few frames. Therefore, occlusions handled properly is very important to CHOG in multiple objects tracking. In this paper occlusions is also predicted by the distance between 
the objects as Ref. 19 and Ref. 20.

$$
\left(E\left(x_{m, k}^{i}\right)-E\left(x_{n, k}^{i}\right)\right)^{2}+\left(E\left(y_{m, k}^{i}\right)-E\left(y_{n, k}^{i}\right)\right)^{2}<R
$$

where $E\left(x_{m, k}^{i}\right), E\left(y_{m, k}^{i}\right), E\left(x_{n, k}^{i}\right)$ and $E\left(y_{n, k}^{i}\right)$ are the mean position of each object, and $R$ is the threshold. When the distance between the object is smaller than the threshold occlusions occurs between them. We can determine which object is occluded by comparing the features of the region with the two objects. After determined the occluded object the weights of which are set to zero in process of the particle filter re-sample. Even though we can not distinguish the occluded objects we can use the GMM to detect them after they pass away from each other. After detecting the object the tracker updates the particles with features, sizes and positions in this region and track it by means of $\mathrm{CHOG}$ particle filter.

\section{Experiments}

In order to test our proposed algorithm, we carried out experiments to track multiple objects in indoor and outdoor image sequences. The experiments are implemented on Pentium(R) Dual with $2.0 \mathrm{GHz}$ CPU and 2.0GB RAM. The indoor image sequence are captured by Logeth camera with $320 \times 240$ pixels per image. The features of each object is composed of color histogram which is calculated in HSV space with $8 \times 8+8$ bins and HOG with 8 bins which is turned around according to its first principle and second principle components. In each frame HOG is calculated with the gaussian smoothing standard deviation $\delta=0.5$ and gaussian weighting of histogram standard deviation $\delta=1$. In Eq.(18) the parameter of $R$ is selected according the height, weight and the scale of the object.

In the first experiment the object $\mathrm{A}$ is occluded by the other. According to Eq.(18) we set the threshold:

$$
R=\sqrt{(A)^{2}+(B)^{2}}
$$

where $A=0.5 W_{n} * S_{n}+0.5 * W_{m} * S_{m}, B=0.5 H_{n} *$ $S_{n}+0.5 H_{m} * S_{m}, W_{n}, H_{n}$ and $S_{n}$ are the mean of the object A's width, height and scale, while $W_{m}$, $H_{m}$ and $S_{m}$ belong to another one. If the the predict distance is lower than $R$ the occlusion occurs.
The color histogram of the objects is similar to each other. If we want to identify which one is occluded we will only depend on the HOG of the objects. In this situations the HOG composed of 72 bins is calculated in the object region according to the estimate position and scale. After determined the occluded object samples of it are not re-sampled since they are meaningless. In this situation particle filter is used to track only one object while the features of the other is reserved. We use the GMM to detect the regions of objects locate. The reserved features are used to determine whether the object is the same to the tracked or not before occlusion. Fig.5 shows the results of the occlusion handling.

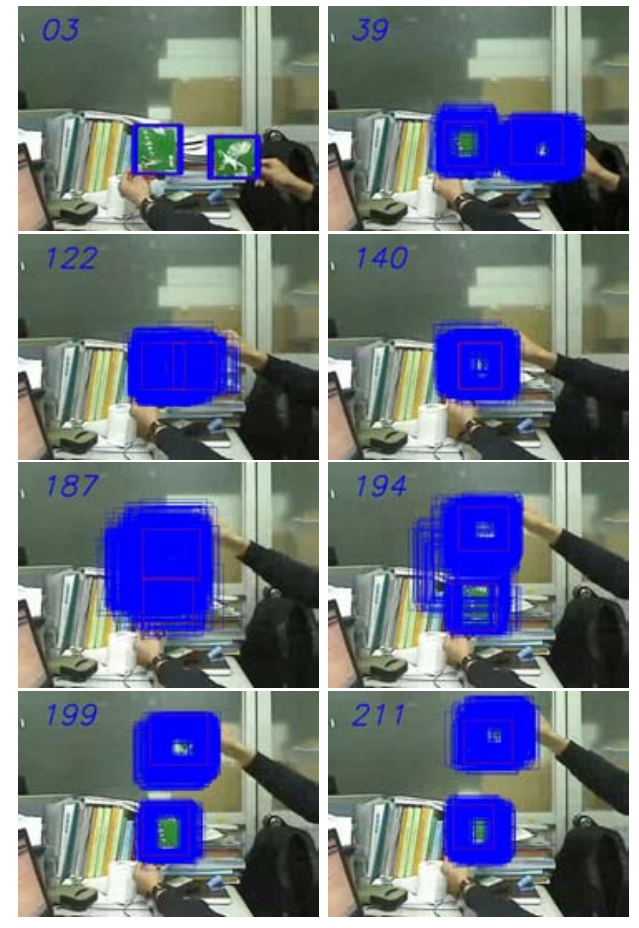

Fig. 5. Two Objects tracking with Occlusion handling.

In the second experiment the aim is to detect and track two objects in cluttered background. The sequence is 500 frames in which two people appearing in the first frame and they are chose as the tracking object. The tracker uses model update particle filter based on CHOG to realize object tracking. The third people appears at $40 t h$ frame, the detector based on GMM detect it in frame 40th but it is only a part. 
After detecting the people we extract the features in object region and initialize the parameters of the particle filter. Along with the whole body of the people appearance in the scene the model update mechanism will obtain the more accurate features step by step. If we get the features of the tracking object beforehand, we will enhance the robustness of the tracker. Comparing with the detection method in Ref. 20 our method can detect the object fleetly. Fig.6 shows the results of object detection and tracking. Fig. 7 shows the results of object detection based on GMM. In Fig. 6 when the object enters the scene two regions including the object are detected. We use the known information of the tracking object to identify the newer. As Fig.6 shows when the object leaves the scene the particles about this object should be give up to save the computational resource.
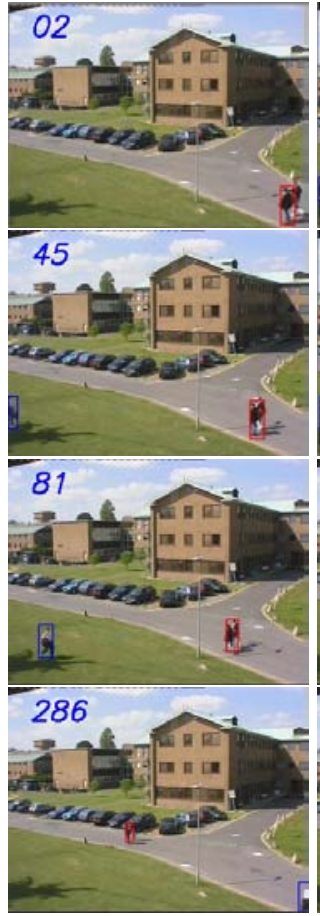

Fig. 6. Object detection and tracking.

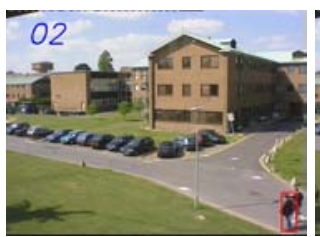

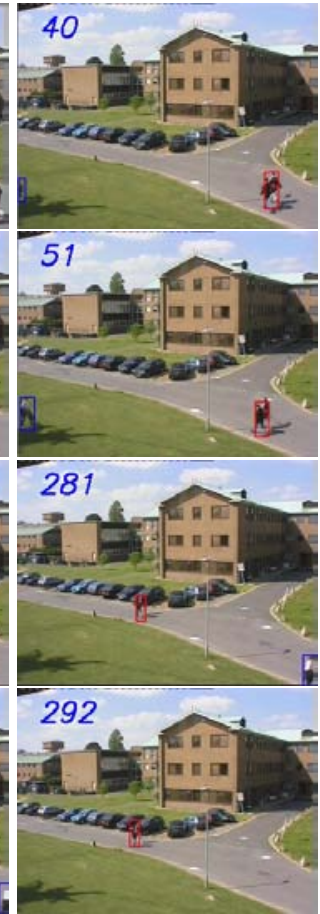

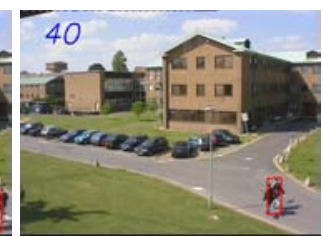

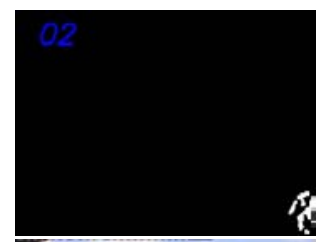
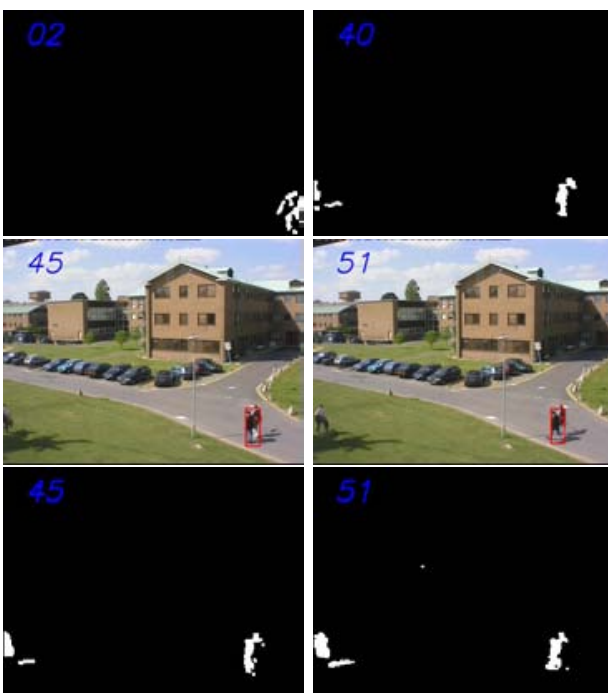

Fig. 7. Object detection results based on GMM.

In the third experiment, the aim is to detect and track footballers in a football sequence. Because of the background model construction based on GMM needs a few frames so the tracking object is selected at frame 10 in our experiment. In frame 10th the footballer of Barcelona with blue and red belt shirt is chose as tracking object. After that the features and position of the footballer are used to initialize the particle filter. The regions including objects are detected in frame 11, after that the features of the objects selected in frame 10 are used to determine which region includes footballer of Barcelona. Even though some existing objects in frame 11 are not detected in time, they will be detected in next frames. In frame 12 we find that a lot of regions may include footballers of Barcelona, we use the particle filter's results to identify the regions which include the tracking object. We only need to compare the features of the unknown regions with the features of the barcelona footballer to determine whether the region includes the object or not. After the tracking object is selected we use the model update particle filter to track it, when the object leaves the scene the corresponding particles should be give up. The results of multiple objects detection and tracking are shown in Fig.8. As Fig.8 shows in frame 10 we choose a Barcelona footballer as the tracking object and calculate the $\mathrm{CHOG}$ as features. The 
accuracy of the tracking algorithm is relate to the number of particles in particle filter. If we choose more particles, the tracking results is more accuracy. But the algorithm has bigger computational requirements with the number of particles increasing. 400 particles are chose in our experiment. When the frame 11 is coming objects similar to the selected in frame 10 are also the tracking object. After the newer objects are determined the initialization of the particle filter is done based on the position, size and features. Frames 10 and 11 show that object detection is real-time sometimes, this is faster than Ref. 20 as Fig.9 shows. Object detection also depends on the background model. Frames 15 and 16 show the tracking results of one object leaves the scene and the other enters the scene. Frames 15 and 16 show the tracking results of one object leaves the scene and the other enters the scene. According to the tracking results and the ratio between the number of objects detected accurately and the number of reality we find that our propose algorithm can detect similar objects and track them with the model adaptive updated.
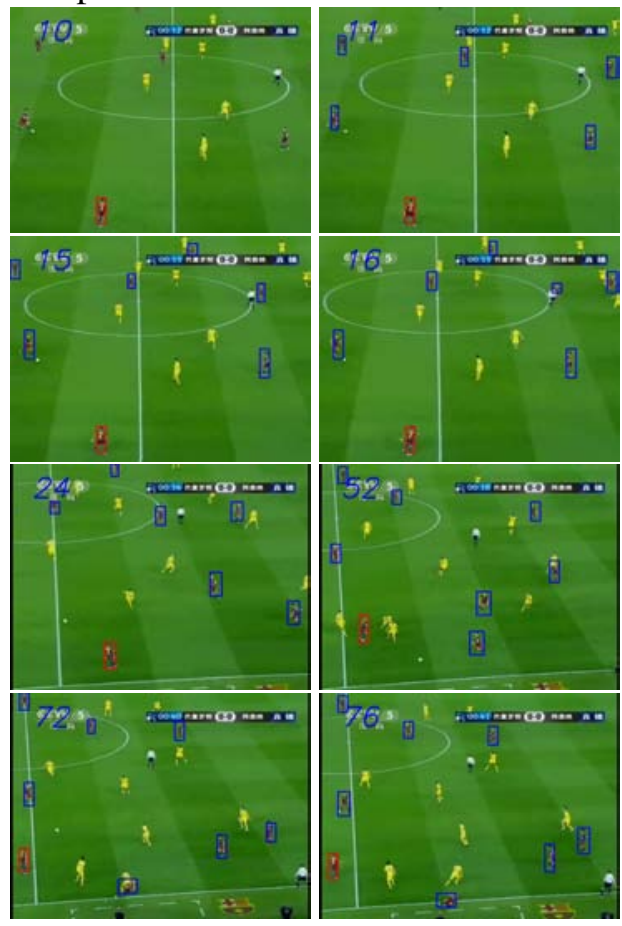

Fig. 8. Multiple objects tracking results.

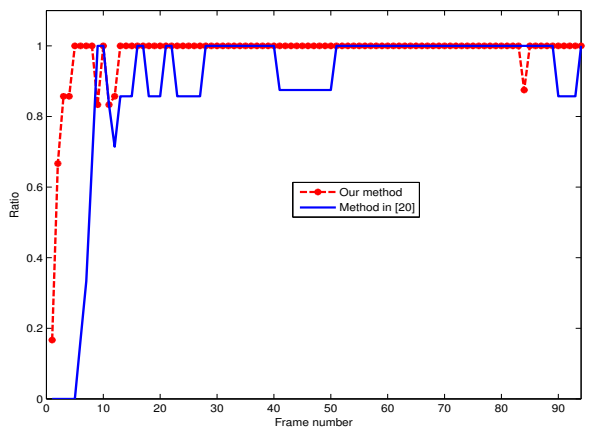

Fig. 9. The ratio between the number of objects detected accurately and the number of reality

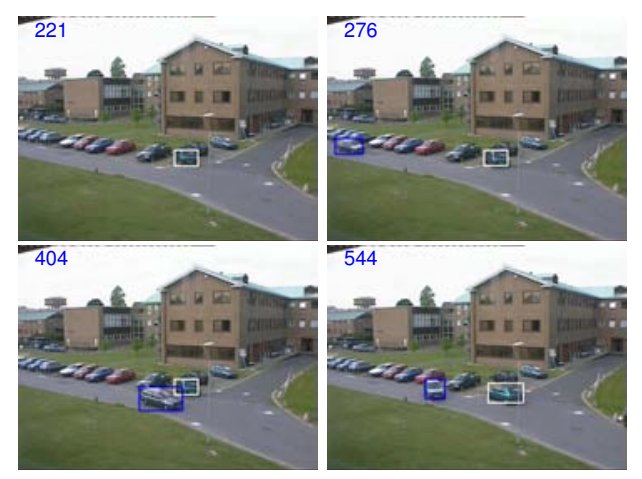

Fig. 10. Results of vehicles detection and tracking.

\section{Applications}

Traffic management and vehicle information rely on a suite of sensors for estimating traffic parameters. To monitor the traffic condition and provide correct vehicles information is important in nowadays due to heavy traffic volume in our daily life ${ }^{31}$. In Ref. 31 the author summarizes the vehicle detection methods. In this section the algorithm is also used in intelligent traffic surveillance System.Firstly, GMM is used to detect the vehicles, after the vehicles are detected we use the $\mathrm{CH}$ and $\mathrm{CHOG}$ of them to initialize the particle filter to realize the object tracking. At the same time the number of the car is update to realize traffic flow control.If the car disappears the tracking about this object is give up. The scale of the vehicle will be changed as it is in different location. So the model update scheme is necessary in 
vehicle tracking. The results of vehicles detection and tracking is as Fig.10 shows.

\section{Conclusion}

This paper presents a novel particle filter for tracking multiple objects. We integrate the $\mathrm{CH}$ and $\mathrm{HOG}$ of the objects together according to the mutual complementarities of the two types of features. In process of tracking the features of the object may slow change so we introduce the kalman filter and GMM to do the features adaptive update. We use the background model based on GMM to do object detection. Particle filter based on CHOG features, model adaptive update and object detection can track multiple objects. The performance of the algorithm is tested on several indoor and outdoor sequence. The results demonstrate the algorithm can detect and track multiple objects successfully. This algorithm can also handle the object occlusion.

A known drawbacks of the algorithm is that objects detection is subject to the precision of the background model based on GMM. The GMM can not cope with large changes of background in time. The drawbacks of the algorithm should be considered in the future work.

\section{References}

1. Puri A, Valavanis K P, Kontitsis M, "Statistical profile generation for traffic monitoring using real-time UAV based video data," Mediterranean Conference on Control and Automation, Athens:IEEE,1-6 (2007).

2. Comaniciu D, Ramesh V, Meer P, "Real-time tracking non-rigid objects using mean shift," IEEE conference on computer vision and pattern recognition 2000 Proceedings, Hilton Head Island, SC, USA: IEEE. 2,142149 (2000).

3. Alper Yilmaz,Omar Javed and Mubarak Shah,"Object Tracking: A Survey,"ACM Computer Surveys, 38(4), 229-240 (2006).

4. Collins R T. Mean-shift blob tracking through scale space. IEEE Computer Society Conference on Computer Vision and Pattern Recognition. Madison, Wisconsin USA: IEEE, 2,230-240(2003).

5. Comaniciu D, Ramesh V, Meer P. Kernel-based object tracking. IEEE Transactions on Pattern Analysis and Machine Intelligence, 25(5),564-575(2003).
6. Junqiu Wang, Yasushi Yagi.Integrating Color and Shape-Texture Features for Adaptive Real-Time Object Tracking.IEEE Transactions on Image Processing,17(2), 235-240(2008).

7. Birchfield S T, Sriram Rangarajan. Spatiograms Versus Histograms for Region-Based Tracking. IEEE Computer Society Conference on Computer Vision and Pattern Recognition,2, 1158-1163(2005).

8. Navneet Dalal, Bill Triggs.Histograms of Oriented Gradients for Human Detection. IEEE Computer Society Conference on Computer Vision and Pattern Recognition , 1, 886-893(2005).

9. Zhenjun Han, Qixiang Ye and Jianbin Jiao.Combined feature evaluation for adaptive visual object tracking.Computer Vision and Image Understanding, 115(1):69-80(2011).

10. Wei-Lwun Lu, Kenji Okuma and James J. Little.Tracking and recognizing actions of multiple hockey players using the boosted particle filter. Image and Vision Computing, 27,189-205(2009).

11. P Viola, M J Jones, and D Snow. Detecting pedestrians using patterns of motion and appearance. The 9th ICCV, Nice, France, 1,734-741(2003).

12. Ning Song Peng, Jie Yanga and Zhi Liu.Mean shift blob tracking with kernel histogram filtering and hypothesis testing.Pattern Recognition Letters, 26(5),605-614.

13. Comaniciu D, Ramesh V, Meer P. The variable bandwidth mean shift and data-driven scale selection. Eighth IEEE International Conference on Computer Vision, Vancouver, BC , Canada: IEEE,1,438445(2001)

14. Hanzi Wang, David Suter, Konrad Schindler and Chunhua Shen. Adaptive Object Tracking Based on an Effective Appearance Filter. IEEE Transaction on Pattern Ananlysis and Machine Intelligence,29(9),16611667(2007).

15. Peter Dunne, Bogdan Matuszewski.Choice of similarity measure, likelihood function and parameters for histogram based particle filter tracking in CCTV grey scale video.Image and Vision Computing,29,178189(2011)

16. Katja Nummiaroa, Esther Koller-Meierb and Luc Van Gool.An adaptive color-based particle filter.Image and Vision Computing, 21,99-110(2003).

17. Hongwei Li, Yi Wu and Hanqing Lu. Visual Tracking Using Particle Filters with Gaussian Process Regression.Lecture Notes in Computer Science, Advances in Image and Video Technology,5414,261-270(2009).

18. Zulfiqar Hasan Khan, Irene Yu-Hua Gu and Andrew G. Backhouse.A Robust Particle Filter-Based Method for Tracking Single Visual Object Through Complex Scenes Using Dynamical Object Shape and Appearance Similarity.Journal of Signal Processing Systems, Online First, 9 October 2010. 
19. Budi Sugandi, Hyoungseop Kim, Joo Koi Tan and Seiji Ishikawa.A color-based particle filter for multiple object tracking in an outdoor environment.Artificial Life and Robotics, 15(1),41-47(2010).

20. Jacek Czyz, Branko Ristic and Benoit Macq.A particle filter for joint detection and tracking of color objects.Image and Vision Computing, 25,12711281(2007).

21. P Prez, C Hue, J Vermaak and M Gangnet.ColorBased Probabilistic Tracking. European Conference on Computer Vision,661-675(2002).

22. David G Lowe.Distinctive Image Features from ScaleInvariant Keypoints.International Journal of Computer Vision,60(2),91-110(2004).

23. Ning Song Peng,Jie Yang,Zhi Liu. Mean shift blob tracking with kernel histogram filtering and hypothesis testing. Pattern Recognition Letters, 26,605614(2005).

24. Nguyen H T, Worring $M$ and van den Boomgaard R.Occlusion robust adaptive template tracking. IEEE International Conference on Computer Vision,1,678683(2001).

25. Christopher Richard Wren, Ali Azarbayejani, Trevor
Darrell, and Alex Paul Pentland. Pfinder: Real-Time Tracking of the Human Body.IEEE Transaction on Pattern Analysis and Machine Intelligence, 19,780785(1997).

26. Chris Stauffer and W E L Grimson.Adaptive background mixture models for real-time tracking. IEEE Computer Society Conference on Computer Vision and Pattern Recognition,2,246-252(1999).

27. Jayesh H Kotecha,and Petar M Djuri.Gaussian sum particle filtering.IEEE Transaction on Signal Processing,51,2592-2601(2003).

28. Jayesh H Kotecha, and Petar M Djuri.Gaussian particle filtering.IEEE Transaction on Signal Processing,51,2602-2612(2003).

29. Hanzi Wang, David Suter, Konrad Schindler and Chunhua Shen. Adaptive Object Tracking Based on an Effective Appearance Filter. IEEE Transaction on Pattern Analysis and Machine Intelligence,29,16611667(2007).

30. Osama Masoud, Scott Rogers, Nikolaos P Papanikolopoulos. Monitoring Weaving Sections. ITS institute Univ Minnesota Minneapolis,1-6(2001). 